\title{
El aprendizaje cooperativo y sus implicancias en el proceso educativo del siglo XXI
}

\section{Cooperative learning and its implications in the 21st century educational process}

Silvia Maritza Medina Bustamante

Universidad César Vallejo, Perú

iamedina5@hotmail.com

https://orcid.org/0000-0002-0217-2506

Recepción: 18/12/2020 | Aceptación: 12/03/2021 | Publicación: 10/05/2021

Cómo citar (APA, séptima edición):

Medina Bustamante, S. M., (2021). El aprendizaje cooperativo y sus implicancias en el proceso educativo del siglo XXI. Innova Research Journal, 6(2), 62-76.

https://doi.org/10.33890/innova.v6.n2.2021.1663

\section{Resumen}

El presente artículo analiza el aprendizaje cooperativo en estudiantes de educación básica y sus beneficios para mejorar los procesos en el aula y el rendimiento escolar. Se basa en la revisión sistemática de investigaciones con estudiantes de los niveles inicial, primaria y secundaria, resaltando su ventaja frente a los modelos individualistas y competitivos que se utilizan tradicionalmente. La información ha sido tomada de artículos científicos publicados en revistas indexadas en bases de datos como Scielo, Redalyc, Dialnet y Google Académico, publicados en los últimos diez años; para la elegibilidad de los artículos y su posterior análisis se aplicó el protocolo Prisma. Los estudios muestran que la dinámica de cooperación entre los estudiantes desarrolla aprendizajes significativos, debido al tránsito de un estado de inactividad y postura receptiva hacia la construcción de sus conocimientos, interactuando con sus pares, con autonomía, liderazgo, autorregulación, respeto y tolerancia; además de ciudadanos responsables, promotores de una cultura de integración y de paz para el bien común. Los docentes se basan en las teorías socioconstructivistas, planifican y desarrollan sus actividades con elementos de cooperación; son los mediadores que organizan y orientan el proceso de los aprendizajes.

Palabras claves: aprendizaje activo; autonomía; cooperación educativa; liderazgo. 


\begin{abstract}
This article discusses cooperative learning in basic education students and their benefits for improving classroom processes and school performance. It is based on the systematic review of research with students at the initial, primary and secondary levels, highlighting its advantage over the individualistic and competitive models that are traditionally used. The information has been taken from scientific articles published in journals indexed in databases such as Scielo, Redalyc, Dialnet and Google Academic, published in the last ten years; for the eligibility of the articles and their subsequent analysis, the Prisma protocol was applied. Studies show that the dynamic of cooperation between students develops significant learning, due to the transit of a state of inactivity and receptive posture towards building their knowledge, interacting with their peers, with autonomy, leadership, self-regulation, respect and tolerance; in addition to responsible citizens, promoters of a culture of integration and peace for the common good. Teachers are based on sociounion theories, plan and develop their activities with elements of cooperation; are the mediators who organize and guide the learning process.
\end{abstract}

Keywords: active learning; autonomy; educational cooperation; leadership.

\title{
Introducción
}

A lo largo de la historia, la educación ha pasado por diferentes etapas que han facilitado su evolución. Hace algunas décadas, las actividades las diseñaba y ejecutaba el maestro, quien pretendía que el estudiante, de manera individual, le demostrara sus aprendizajes, fundamentalmente conocimientos basados en la memoria. En los últimos años, esta situación ha cambiado notablemente, lo cual, obliga a los maestros a enfrentar grandes desafíos por la rápida producción de nuevos conocimientos, la revalorización de las diversas culturas, el mayor empleo de los entornos virtuales y de las TIC, en un contexto en donde el individualismo y el espíritu competitivo aún perduran, pero con menos eficacia, en las escuelas. De ahí, el valor de implantar en el proceso de enseñanza aprendizaje, la cultura de cooperación que incentive la importancia y necesidad de trabajar juntos en interacción, a partir del apoyo, ayuda mutua, en un ambiente favorable, de respeto y construcción de conocimientos.

El aprendizaje cooperativo, aunque no es algo novedoso, toma relevancia en el campo educativo, como una metodología activa en el que, el éxito del estudiante depende de que logre la meta establecida (Lobato, Guerra y Apodaca, 2015) respetando sus particularidades y ayudándolos a desarrollar sus potencialidades en interacción cooperativa con sus pares. Las investigaciones en el campo de la educación y psicología sustentadas en teorías, permiten evidenciar de manera constante la superioridad y efectividad del aprendizaje cooperativo, en relación al planteamiento de métodos tradicionales o aquellos en el que solo se brindan situaciones para el aprendizaje individualista o competitivo (Mayordomo y Onrubia, 2016)

Estas consideraciones permiten determinar como propósito para esta investigación, evidenciar los beneficios del trabajo cooperativo en el aprendizaje de los estudiantes, con la puesta en práctica de un rol docente activo, que conciba a la educación como el proceso en el que el aprendizaje debe gestionarse a partir de la interacción de quienes intervienen en ella como parte importante en el desarrollo humano, pues ello permitirá favorecer la integración, respeto a la 
diversidad, desarrollo socio afectivo, la motivación, entre otros aspectos que se abordarán y que permitirán determinar entre otras ventajas, que una metodología activa como el aprendizaje cooperativo potenciará la práctica docente y mejorará sustancialmente el logro académico de los estudiantes, como lo determinan las investigaciones referidas, así como las diversas teorías del aprendizaje que sustentan el valor de esta estrategia didáctica.

\section{Desarrollo}

Orientar el modelo educativo que optimice la aplicación de metodologías hacia el logro significativo de los aprendizajes y la formación integral de los estudiantes, requiere comprender que ello se refiere a una verdadera educación de calidad. Para que así ocurra, es necesario considerar y establecer políticas, mecanismos y estrategias que permitan potenciar el saber pedagógico de los docentes, a fin de lograr la eficacia en su trabajo, que posibilite, a partir de ello, aprendizajes valiosos en sus estudiantes.

El actuar educativo, sustentado en políticas y documentos normativos, conciben a la educación como un derecho a partir de la Declaración Universal de Derechos Humanos de las Naciones Unidas en 1948; posteriormente, la Convención de los Derechos del niño en 1989 y así a lo largo del tiempo, considerándose aún como un reto, generar en todos los estudiantes aprendizajes significativos con oportunidades para todos, que les permitan desarrollar su máximo potencial; en el año 2000 se consideraron los Objetivos de desarrollo del milenio (ODM) y el programa de las Naciones Unidas, de Educación para todos (EPT) hasta el año 2015 (Cueto, 2016). Posteriormente, la UNESCO, con el diseño de la agenda 2030 y los objetivos de desarrollo sostenible, aprobada en el año 2015 en Asamblea General de las Naciones Unidas, dan prioridad en la hoja de ruta, a la educación inclusiva y de calidad con oportunidades permanentes para todos en los distintos niveles. En el Objetivo 4, precisa que la educación de calidad está garantizada, considerando la equidad y la inclusión, buscando brindar situaciones para que el aprendizaje se desarrolle de manera continua. La meta 4.1 es lograr que todos los estudiantes terminen con una educación de calidad de manera equitativa y gratuita con aprendizajes significativos y acertados. La meta 4.7 plantea, entre otros, el promover a través de la educación, el valorar la diversidad cultural, los derechos humanos y la cultura de paz. (Naciones Unidas, CEPAL, 2018)

En Perú, la Ley General de Educación, en su artículo 2, considera que la educación permite que las personas se formen de manera integral en un proceso de enseñanza y aprendizaje a lo largo de toda la vida; además, en el artículo 3 precisa que las personas tienen derecho a acceder a ella, con calidad. (Ministerio de Educación, 2003). Por ello, el Proyecto Educativo Nacional (PEN) al 2021, en el objetivo estratégico 2 plantea promover el logro pertinente y de calidad de los aprendizajes de estudiantes e instituciones educativas, lo que implica lograr las competencias requeridas para la formación integral de los estudiantes, con aprendizajes significativos (Consejo Nacional de Educación, 2006). En esa línea, las aspiraciones educativas expresadas en el perfil de egreso, contenido en el Currículo Nacional, señalan que deben desarrollarse competencias que permitan a los estudiantes, según sus características, intereses y aptitudes particulares, participar de manera empática, asertiva, ética y tolerante, trabajando colaborativamente hacia objetivos comunes, regulándose emocionalmente y en sus comportamientos, demostrando habilidades para 
resolver conflictos y desarrollar el trabajo en equipo, tomando decisiones con autonomía, cuidando de sí mismo y de los demás. (Ministerio de Educación, 2016).

De esta forma, les corresponde a los maestros comprometerse con sus estudiantes y sus aprendizajes, considerando las diversas necesidades familiares, sociales, culturales y ambientales que le permitan aportar al desarrollo de su potencial, construyendo vínculos afectivos positivos (Consejo Nacional de Educación et al., 2020). Al respecto, desde el 2012, el Marco del Buen Desempeño Docente, en la competencia 3, desempeño 14, resalta la importancia de que el docente propicie oportunidades para que los estudiantes realicen trabajos en grupos, ayudándose entre compañeros para lograr aprendizaje con la contribución de todos sus miembros (MINEDU, 2018). Entonces, desde el inicio de la escolaridad, se pretende dar atención a todos los estudiantes, según sus ritmos y estilos de aprendizaje, buscando fortalecer entre ellos, las relaciones de cooperación para el desarrollo de competencias.

\section{Teorías relacionadas con el aprendizaje cooperativo}

Diversos estudios, dentro del campo educativo, destacan la importancia del aprendizaje caracterizado por la cooperación, sentándose las bases y principios de éste en el campo de la psicología social. Thorndike (1938) es uno de los que trabajó investigaciones para determinar si trabajar la resolución de problemas de manera cooperativa era mejor que hacerlo de forma aislada; concluyendo en la superioridad de dos cabezas, frente a una. Unos años después, Deutsch (1949) señaló que en el aprendizaje cooperativo existen dos aspectos básicos, la unidad de meta y la colaboración para llegar a ella entre los que participan, en un proceso de interacción (García, Traver y Candela, 2019).

En este proceso histórico en el que, además, se dan espacios para el aprendizaje competitivo e individualista, cobra mayor interés el aprendizaje cooperativo como metodología activa desde fines del siglo XX a la fecha, por sus aportes en lo académico, psicológico, cognitivo, sociológico y más (Juárez, Rasskin y Mendo 2019). Estas investigaciones fueron trabajadas por (Johnson, Johnson y Holubec 1999) y son tomadas por muchos como referentes, pues con sus aportes, establecieron entre otras consideraciones que, para que se pueda trabajar de manera cooperativa, es necesario identificar elementos o dimensiones que se deben tener en cuenta para que se realice un trabajo cooperativo efectivo y con ello se produzca el aprendizaje.

Los aportes de las teorías del aprendizaje permiten fundamentar la importancia del aprendizaje cooperativo. Así tenemos a Jean Piaget, precursor de la teoría cognitiva, quien desarrolló la tesis de que, el modo de pensamiento de los niños, va cambiando con el tiempo y las acciones que experimenta, señalando que estos procesos del pensamiento tienen influencia en la conducta y que el aprendizaje resulta de la activa participación de la persona en la construcción del conocimiento gracias a las experiencias física e intelectual en un proceso activo que se produce constantemente (Olmedo y Farrerons, 2017). Cuando ocurre la generación de conflictos cognitivos es que surge la necesidad de aprender, llevando a nuestro cerebro a buscar nueva información y explicaciones que conecten con la que estaba almacenada en nuestro cerebro (Bringas, 2018). Por ello, es fundamental la interacción social para potenciar las estructuras intelectuales superiores, generar los conflictos cognitivos y como resultado de la confrontación de las diferentes 
perspectivas de sus integrantes, se logren reestructurar los aprendizajes, encontrando soluciones y asimilando puntos de vista diferentes, llevando ello, a producciones intelectuales de mayor valor.

El paradigma que propone Vygotzky, pionero de la perspectiva sociocultural, se focaliza en el desarrollo humano, que se produce con la interrelación de la maduración orgánica con la historia cultural. Sostuvo que cada persona desarrolla competencias que aprende de otras, dentro de la sociedad en la que se desenvuelve y los diferentes grupos en los que interactúa (Gallegos, 1997). Su aporte fundamental es el de la zona de desarrollo próximo, en el que cada estudiante aprende una gama de aspectos relacionados con su nivel de desarrollo, existiendo otros que solo pueden ser interiorizados con el apoyo de un adulto o con un par más destacado, esta interacción permitirá que, al aprender un día con la ayuda de alguien, pueda hacerlo luego por sí solo. Sostiene, además, que el lenguaje es el medio que permite el desarrollo de diversas habilidades mentales como la memoria, atención, concentración; así también, propone la necesidad de la "mediación" como el "puente" para que las estructuras mentales se modifiquen y se pueda llegar al nuevo conocimiento, siendo indispensable para que se produzca el aprendizaje, la interrelación entre las personas y el ambiente (Bringas et al., 2018). El aprendizaje cooperativo permite que el lenguaje se vea fortalecido y promueva entornos favorables al brindarse la ayuda y apoyo de manera mutua.

Ausubel considera que el aprendizaje se da en un proceso en el que se relaciona información nueva con algún aspecto que ya existe en la estructura cognitiva de una persona, de relevancia para el material que se quiere aprender, cuando es capaz de darle a un contenido el significado, se construye el aprendizaje (Guerrero, 2014). Este, en tanto sea relevante, se relacione con su entorno, cultura, ambiente, despertará su interés, será significativo para el estudiante, tendrá sentido y no se le olvidará fácilmente, considerando como un factor importante del aprendizaje el conocimiento previo que poseen los estudiantes para obtener otros nuevos (Bringas, et al., 2018). El aprendizaje cooperativo promueve los aprendizajes significativos al permitir interacciones donde pueden asociar las ideas que ya tienen con las nuevas que obtienen de sus compañeros, logrando procesar y comprender mejor los conocimientos, generando satisfacción, interés por aprender, nuevas estrategias de aprendizaje en un clima de trabajo positivo que el docente debe motivar para la activa participación de los estudiantes (Díaz y Hernández, 2015).

\section{Aprendizaje cooperativo}

En el mundo globalizado en que vivimos, la educación requiere potenciar prácticas que estimulen la convivencia entre las personas con diferentes habilidades, características socioculturales, académicas; en ese sentido, el ejercicio pedagógico que promueve el aprendizaje cooperativo desde temprana edad, ayuda en la formación integral de los estudiantes. Esta metodología, según La Prova (2017), nace por los años 70 en Estados Unidos ante la necesidad de atender en el ámbito educativo, situaciones que lleven a la socialización y a una saludable convivencia, siendo así como, a través de los años, se trabajan las competencias para ello, debido a que son necesarias las habilidades interpersonales para la cooperación en el trabajo grupal, el aprendizaje y la responsabilidad con respecto a otros compañeros (Martínez y Sánchez, 2020).

Se parte de la idea de que el aprendizaje, según Sáez (2018) es considerado como el proceso en que una información es asimilada, observándose un cambio en el comportamiento. Para que se produzca y sea significativo, deben considerarse, la necesidad de aprender, la motivación, la 
preparación para el aprender, entre otros, mediante un proceso de construcción personal y grupal, participando directamente y de manera activa. Supone un cambio en las representaciones a nivel mental obtenido como resultado de la experiencia. (Heredia y Sánchez 2020) El término cooperar se entiende como el trabajo desarrollado en equipo para lograr objetivos comunes que generen beneficios a nivel personal y grupal.(Johnson et al., 1999).

El aprendizaje cooperativo es una estrategia educativa que se está potenciando en las últimas décadas, pues se le considera como la herramienta pedagógica que permite atender las necesidades de las personas del presente siglo (Azorin 2018), enseña a los estudiantes a trabajar de manera conjunta y organizada, en pequeños grupos mixtos para desarrollar actividades académicas, profundizando en su propio aprendizaje con reflexiones que dejan de lado aspectos individualistas y competitivos, abriendo espacios a una práctica en la que se optimiza el aprendizaje propio y el de los demás (Johnson et al., 1999). Esta forma de organización permite a los estudiantes darse cuenta de que pueden aprender y lograr sus objetivos si sus compañeros también lo hacen (Mayordomo y Onrubia, 2016).

García, Traver y Candela, (2019) consideran que el aprendizaje cooperativo se produce cuando se estructura el conocimiento en un proceso en el que las mentes se van adecuando con una interacción entre profesor-estudiante y entre pares. Ello permite una influencia recíproca a partir del intercambio de pensamientos, sentimientos, reacciones, a través de un espacio comunicativo en el que, mediante esa comunicación, se busca alcanzar los objetivos. Por ello, estas relaciones deben darse en un clima de respeto y cooperación, con estudiantes que puedan tomar decisiones a partir de la colaboración y confianza, que apertura el deseo de aprender y estructurar aprendizajes significativos.

El trabajo en equipo, por sí solo, no asegura un aprendizaje cooperativo, Johnson y Johnson (2015) precisan cinco elementos o también llamadas dimensiones que son necesarias para que se produzca:

Interdependencia Positiva, los integrantes de un grupo ante una tarea propuesta, reconocen que el beneficio del esfuerzo que realicen será personal y grupal, lo que les generará un compromiso con el éxito de los demás y el propio, constituyéndose en la base del trabajo cooperativo.

Responsabilidad individual y grupal, donde cada estudiante se responsabiliza en cumplir con la parte del trabajo asignado, asumiendo el compromiso para el logro del objetivo evaluando el progreso del trabajo de acuerdo a éste, así como los esfuerzos individuales para identificar las fortalezas y necesidades de cada uno, dar ayuda y respaldo, aprendiendo juntos.

Interacción estimuladora, los estudiantes desarrollan juntos una tarea, promoviendo cada uno el triunfo de los demás, ayudándose, resguardándose, felicitándose por el esfuerzo que realizan para lograr aprender, conectando sus aprendizajes presentes con los que ya tenían.

Prácticas interpersonales y grupales, para que el trabajo en equipo se optimice, sepan cómo desempeñar la dirección y tomar decisiones, manejando adecuadamente los problemas para el buen funcionamiento del grupo, comunicándose en un clima de confianza y de motivación.

Evaluación grupal, analizando en qué medida van alcanzando sus metas, en relación a un trabajo eficaz, reconociendo las acciones positivas y negativas para la toma de decisiones sobre aquello que deben conservar o cambiar, valorando el trabajo juntos hacia la mejora, para que el 
grupo sea más eficaz. Se precisa, además, que en los grupos de aprendizaje cooperativo se busca un mejor desempeño a nivel individual como respuesta al aprendizaje logrado en grupo.

\section{Relevancia del aprendizaje cooperativo en el aula}

Los desafíos que nos presenta el siglo XXI conllevan a revalorar la efectividad de la cooperación ante los retos mundiales que conducen a una interdependencia global a nivel tecnológico, económico y ambiental que requieren enfrentarlos, viviendo en democracia, respetando la diversidad con relaciones interpersonales positivas que efectivicen el manejo de conflictos, con ciudadanos emprendedores, creativos, innovadores capaces de interactuar en diversos entornos y medios. Ante ello, la revisión de diversos artículos de investigación y tesis corroboran el impacto positivo y satisfactorio de su aplicación en los diferentes niveles y modalidades de la escolaridad para el desarrollo de conocimientos, habilidades, valores y actitudes, que requiere para enfrentarse de manera competente a la realidad cambiante de nuestros tiempos de empleo de tecnología, relaciones virtuales y necesidad de generar entre todos la cooperación y el esfuerzo coordinado para optimizar el contacto entre las personas y hacer frente a los desafíos de manera satisfactoria (Johnson y Johnson, 2014).

Pevida, Dolores y Gallego (2014), a partir del desarrollo de un proyecto intercentros en Madrid, España, cuyo objetivo era desarrollar cambios educativos para el éxito personal y académico de los estudiantes mejorando el trabajo en equipo concluyen que el aprendizaje cooperativo ayudó a los estudiantes a ser colaboradores, tolerantes, solidarios, autónomos, creativos y competentes, motivándose en ellos, el deseo de superarse, integrando la diversidad existente en las aulas. Así también, León, Mendo e Iglesias, (2015) afirman, que el Aprendizaje Cooperativo interviene en las habilidades sociales del grupo considerándolo como base para desenvolverse de manera eficaz en las diferentes situaciones de trabajo cooperativo, con resultados de mayor calidad, como también señalan Cifuentes y Meseguer (2015).

Sánchez y Casal (2016), en su artículo referido al desarrollo de la autonomía a partir de técnicas para el aprendizaje cooperativo, concluyen que éste logra tres aspectos esenciales: reduce la ansiedad, aumenta la motivación y permite atender los diversos estilos de aprendizaje. Destacan que estos aspectos, posibilitan en los estudiantes, sentir satisfacción al buscar y encontrar estrategias para lograr los objetivos propuestos, llevándolos a comprenderse mejor con respecto a sus fortalezas y debilidades, así como a interactuar con los demás asumiendo actitudes positivas. Se añade a ello, el estudio desarrollado por García-Cuevas y Hernández (2016) en el que afirman que el aprendizaje cooperativo también es de gran utilidad en la inclusión de estudiantes con trastorno de espectro autista.

Soto (2017) concluye que el trabajo colaborativo ayuda a socializar e interiorizar los conocimientos en los estudiantes e influye en su rendimiento académico, recomendando fortalecer la integración en el aula para potenciar la inteligencia emocional. Por otra parte, esta metodología se puede trabajar a través del internet como lo demostró en su investigación Torres, (2017) evidenciando que los recursos de la red, tanto visuales como auditivos, motivan y favorecen el aprendizaje de idiomas extranjeros como el inglés. En el 2017, Huanca determinó que tiene efectos significativos en el progreso de los dominios de matemática en los estudiantes de quinto de primaria precisando que la interacción social permite que los conocimientos sean útiles al ser 
compartidos en el trabajo en equipo con objetivos comunes. Más adelante, Herrada y Baños (2018) amplian la idea, considerándolo como un método conveniente para enseñar matemática en cualquier etapa escolar, mientras que Morales, García, Torres y Lebrija, (2018) agregan que permite el logro de aprendizajes, la integración y desarrollo de capacidades, comportamientos y conocimientos, mejorándose las relaciones interpersonales y el logro, a través de la enseñanza recíproca entre pares, de aprendizajes de calidad.

Los aspectos teóricos desarrollados sustentan en los resultados de las investigaciones, los beneficios de la aplicación de la metodología abordada y en esa orientación se recoge el aporte de García, et al. (2019) para entender la relevancia de un aprendizaje cooperativo y la promoción de éste en el proceso educativo. Ellos destacan la prevalencia de una interdependencia positiva en la que todos los integrantes del grupo se interesan por el aprendizaje que va logrando cada uno, a fin de que potencie al máximo el rendimiento. Las relaciones e interacciones entre los estudiantes permiten el aprendizaje de contenidos académicos, así como habilidades y conductas sociales que fortalecen la autoestima, empatía, integración, identidad, desarrollo social, capacidad para soportar tensiones y adversidades. Por ello, Johnson y Johnson (2015) señalan la necesidad del aprendizaje cooperativo para que los estudiantes adquieran valores, patrones de comportamiento y actitudes necesarios para su desenvolvimiento efectivo en democracia.

Los grupos se constituyen con estudiantes de diversas características, ritmos, estilos de aprendizaje, consolidándose grupos heterogéneos, lo que genera condiciones para la aparición de conflictos socio-cognitivos, apareciendo en esa búsqueda de respuestas, opiniones diferentes y el aumento de una actividad cognoscitiva entre ellos, con la estructuración de significados y conocimientos compartidos (Johnson y Johnson, 2015). Los esfuerzos desarrollados para obtener buenos resultados les generan un mejor rendimiento y mayor productividad, retención a largo plazo de lo aprendido, razonando en un nivel superior y desarrollando el pensamiento crítico, con una comunicación asertiva, interdependencia y responsabilidad que permite al equipo desarrollar con éxito las tareas asignadas (León, Felipe, Iglesias y Marugán, 2014).

Se establecen relaciones positivas entre los estudiantes, fortaleciéndose el compromiso en equipo, relaciones de tolerancia, respeto y solidaridad, valorando la diversidad (Johnson et al. 1999). Estas emociones favorables, como señala Ibarrola (2014) favorecen los procesos de aprendizaje, auto-motivando a los estudiantes, elemento indispensable en la mejora del rendimiento; potencia el liderazgo compartido, cuya meta, en cada actividad, se enfoca en realizar la tarea solicitada y que, al término de ella, se produzca el mayor aprendizaje posible. Zariquiey (2016) enfatiza que esta metodología permite aprovechar a través de la interacción social, las potencialidades de cada integrante independientemente del nivel en que se encuentren. Los estudiantes organizan su tarea, se responsabilizan en el desarrollo de su parte, liderando en este aporte y compartiendo con los demás, evidenciándose un alto grado de igualdad en los roles y un potencial de ayuda que permite la interacción efectiva entre sus integrantes.

Esta metodología activa, basada en la cooperación para el desarrollo de diversas tareas, favorece a los estudiantes en el desarrollo o potenciación de las diversas inteligencias sustentadas por Howard Gardner, acrecentando las posibilidades de éxito entre todos los estudiantes debido al incremento de experiencias educativas estimuladas en el trabajo cooperativo. Las interacciones establecidas permiten el desarrollo del lenguaje, así como de la inteligencia interpersonal, 
evidenciándose en la escucha activa, habilidades sociales, empatía, manejo de conflictos con sus pares (Torrego et al. 2011).

La mirada educativa actual, considera como centro al estudiante que aprende en un proceso de participación, interacción y cooperación, en concordancia con la dinámica de nuestros tiempos; por ello, está obligada a promover el empleo efectivo y pertinente de la tecnología así como de los entornos virtuales en la estrategia de aprendizaje a distancia de manera sincrónica o asincrónica, optimizando el rol activo y protagónico de los estudiantes a través del aprendizaje cooperativo, desterrando las diferencias culturales con las diversas formas de comunicación que se ofrecen, permitiendo al docente asumir el rol mediador para la gestión de los recursos y las posibilidades de trabajo en equipo, con un modelo educativo orientado no solo al logro de productos académicos, sino también a la mejora de las relaciones interpersonales y el aprendizaje a través de la cooperación (Rodríguez y Barragán, 2017), competencias fundamentales en una sociedad que requiere ciudadanos empáticos, críticos, reflexivos, con capacidad de discutir ideas en los diversos espacios en que se desenvuelve de manera interactiva.

\section{El docente y la metodología cooperativa en el aula}

Incorporar el aprendizaje cooperativo como metodología activa dentro del aula no es tarea sencilla, el maestro no se limita a una revisión del producto, interviene de manera directa, monitoreando el trabajo que va desarrollando el equipo en un modelo de enseñanza inclusivo. Mendo, (2019) resalta la importancia de brindarle a los estudiantes estrategias y herramientas que aseguren éxito en las acciones de cooperación e interacción social. En esa dirección, se hace necesario que el docente fortalezca sus competencias para ser agente de cambio en los estudiantes, ofreciéndole oportunidades para su formación plena e integral, con herramientas para el desarrollo de su pensamiento complejo, humano y desarrollo de sus competencias sociales (Buenfil, Alvarado y Barba, 2019), trabajando en el aula aprendizajes de manera cooperativa, creando un ambiente favorable para facilitar relaciones de reciprocidad, solidaridad, diálogo y confianza (Robles, 2015).

Desarrollar una práctica docente desde la perspectiva de un trabajo cooperativo requiere de una constante reflexión y formación para llevar a los estudiantes a aprender a aprender (Serrano y Pons, 2014) como también lo señalan Lata y Castro (2016), resaltando la necesidad de una práctica reflexiva individual constante para su desarrollo profesional y mejora de su trabajo en aula, creando un clima de trabajo de alegría y sociabibilidad, con relaciones interpersonales afectivas, estados de motivacion e interés por aprender junto a sus pares y docente (Slavin 1999).

Johnson et al. (1999) consideran necesario que los docentes puedan estructurar las sesiones cooperativamente, con el establecimiento de los objetivos conceptuales y actitudinales en la planificación de la sesión, decidiendo los materiales que emplearán y la forma como organizarán a los equipos en el aula. Así, cada práctica debe pasar por ese proceso de reflexión y evaluación sobre cómo funcionó, qué se podría implementar para mejorar la cooperación entre los estudiantes, teniendo como criterios las dimensiones abordadas, hasta ir adquiriendo, de manera gradual y creciente, la experiencia en esta estrategia compleja de enseñanza. Ayudará al docente, considerar el sustento de la teoría de las inteligencias múltiples, pues con esta metodología cooperativa se puede dar atención a todos los estudiantes, al combinarse el potencial que trae cada uno y que permite un aprendizaje cooperativo procesado y comprendido por ellos. 
Los docentes, en una clase cooperativa, deben tener en cuenta aspectos como los planteados por Johnson et al. (1999):

Selección de objetivos didácticos, precisando los objetivos conceptuales que señalan los conocimientos que los estudiantes aprenderán y las conductas que establecerán a nivel interpersonal para una cooperación eficaz. Así también, los objetivos actitudinales, referidos a las interrelaciones que se establecerán en el trabajo colectivo.

Selección de materiales didácticos, considerándose que la distribución de los mismos promueva cooperación y una interdependencia positiva que les permita trabajar juntos para el cumplimiento de la tarea, como el dar un solo instructivo con preguntas a cada equipo para que sean respondidas de manera conjunta.

Conformación de los grupos, el docente debe tener en cuenta la cantidad de estudiantes por grupo, la distribución de los mismos y el tiempo de duración de los grupos. Con respecto a la cantidad, esta va a depender de la edad y los objetivos de la sesión, siendo lo conveniente entre 2 a 4 integrantes. Se debe considerar que, mientras más grande es el número, las interacciones disminuyen; en un grupo más pequeño, el desempeño de cada estudiante se hace más notorio. La distribución de los estudiantes en los grupos puede hacerse al azar, determinado por el docente o por los mismos estudiantes. La duración de los grupos dependerá del tipo de aprendizaje cooperativo que se quiere lograr, puede durar una o varias sesiones.

Disposición del aula, los integrantes de los grupos deben sentarse mirándose a la cara y con posibilidades de trabajar compartiendo materiales e intercambiando ideas, así como, poder mirar al docente sin dificultad. Entre los grupos, debe existir distanciamiento para que entre ellos no se interrumpan y se pueda caminar con fácil acceso hacia los materiales distribuidos en un aula adecuadamente iluminada y ventilada.

Asignación de roles, para que cada integrante de los grupos conozca lo que se espera de él, con ello se logrará que adopten una participación activa, que entre todos los estudiantes tengan claridad de lo que van a desarrollar.

Ejecución de tareas, el docente explica de manera clara la tarea asignada dando a conocer el resultado que se espera para que los estudiantes se orienten a ello. Se absolverán las preguntas de los estudiantes, mediante ejemplos para que se entienda lo que van a aprender y hacer. Cada integrante de los grupos debe evidenciar lo trabajado.

Evaluación de la calidad y cantidad del aprendizaje, deben establecerse en cada sesión los criterios que serán evaluados, lo que permitirá al docente diagnosticar el trabajo y comprensión de los mismos para la retroalimentación.

Eficacia del grupo, la reflexión sobre el trabajo desarrollado, con la retroalimentación respectiva, optimiza la eficacia de su actuar y el compromiso de mejorar en las nuevas sesiones que desarrollen. Esta reflexión los llevará a precisar las acciones que les permiten el cumplimiento de sus metas para la toma de decisiones con respecto a aquellas que debe modificar, potenciar o 
continuar desarrollando. Es necesario que, en cada grupo, los estudiantes reciban la retroalimentación que los motiven a tomar las acciones respectivas para la mejora de su rendimiento y el establecimiento de nuevas metas. En este procesamiento grupal es necesario que todos participen en la reflexión del funcionamiento del equipo.

El aprendizaje cooperativo, como cualquier otra estrategia, puede presentar dificultades en su desarrollo. Al respecto, Slavin et al. (1999) señala que se pueden superar, atendiendo a la situación que las origina:

Fracaso en llevarse bien, ocurre generalmente durante la conformación de equipos que suele integrar a variados estudiantes de diversas características. Ante ello se sugiere que no se cambie de equipo al estudiante y que se le motive a trabajar contribuyendo en el éxito del mismo. El trabajo en pareja debe combinarse, dentro del equipo, para fijar el entendimiento de la importancia de su formación individual y del equipo en su conjunto.

Mala conducta, la que puede revertirse si se estimula a los grupos con expresiones motivadoras, recompensas por el esfuerzo conjunto, grado de cooperación y conducta adecuada.

Ruido, en un aula de aprendizaje cooperativo debe evidenciarse el sonido de las conversaciones en el interactuar de los estudiantes; la alteración de ello debe dar paso a que el docente haga un alto a la actividad que desarrollan para recordar que deben emplear un tono de voz bajo.

Empleo ineficaz del tiempo, que podría ocurrir cuando los estudiantes se esfuerzan solo por terminar la parte que se les asignó y no se preocupan por los demás miembros de su grupo. Ante ello, se les puede estimular para que trabajen en parejas o de tres una misma actividad, verificando el correcto cumplimiento, apoyando al compañero que tuvo dificultad, asegurándose de que todos entiendan. Esto favorecerá la intención del aprendizaje cooperativo, en buscar no sólo terminar la tarea de manera individual, sino apoyar al compañero del grupo, buscando entender y hacer que todos aprendan.

Nivel diferente de desempeño, trabajar individualmente con aquellos que presentan dificultad de aprendizaje para el apoyo al logro del nivel de los demás.

\section{Conclusiones}

Las normativas internacionales y las de cada país son las que sientan las bases del desarrollo de una educación de calidad acorde a los requerimientos del siglo XXI, orientada a la formación de personas autónomas, capaces de convivir y actuar respetando su individualidad, la de los demás y del entorno; para lo cual, el trabajo cooperativo se convierte en una estrategia fundamental en los diversos espacios educativos.

Las teorías de Piaget, Vygotsky, Ausubel, si bien surgieron hace unas décadas atrás, actualmente, son las más utilizadas en la aplicación del aprendizaje cooperativo, porque promueven las interacciones entre los estudiantes a partir de actividades retadoras y de interés; 
generan conflictos cognitivos; el aprender de manera activa, crítica, responsable y modificando continuamente sus estructuras cognitivas y habilidades sociales al aprender cooperativamente.

El aprendizaje cooperativo contribuye al desarrollo cognitivo y permite que los estudiantes se vuelvan autónomos e independientes en su aprendizaje con respecto a su maestro, promueve el desarrollo del pensamiento crítico, la integración y aceptación a las diferencias interculturales, el desarrollo socio-afectivo y la mejora del rendimiento académico, incluyendo actividades que requieran el empleo de entornos virtuales.

La cultura de cooperación, a lo largo de la vida escolar, facilita la generación de ciudadanos responsables, promotores de una cultura de integración, trabajo en equipo con liderazgos compartidos y reflexión en la toma de decisiones orientados a promover una cultura de paz, en busca del bien común, aspectos fundamentales a trabajarse desde temprana edad ante la demanda de los tiempos que vivimos y en los que es fundamental la formación integral de las personas, con las competencias que les permitan actuar de manera pertinente.

Con el trabajo cooperativo, los maestros despliegan una dinámica de interacción en el aula con equipos de trabajo formado por estudiantes; planifican y desarrollan sus actividades con elementos de cooperación para que ellos se apoyen mutuamente en una interdependencia positiva, asumiendo responsabilidades a nivel individual y grupal en la parte de la tarea asignada; aprendiendo en equipo tanto a comprender como resolver problemas; desarrollando habilidades para la comunicación, liderazgo, confianza, toma de decisiones, solución de conflictos, intercambio de información, análisis y reflexión; autoevaluando el logro de sus metas, problemas o fracasos para los cambios respectivos y la mejora de sus trabajos posteriores.

\section{Referencias Bibliográficas}

Azorín Abellán, C. (2018). El método de aprendizaje cooperativo y su aplicación en las aulas. $\begin{array}{lllll}\text { Perfiles } & \text { Educativos, } & 40(161), & 181 & -\end{array}$ https://doi.org/10.22201/iisue.24486167e.2018.161.58622

Bringas, N. (2018). Hallazgos de la Neurociencia que deberían ser considerados en Educación. (20). 63-67. https://doi.org/10.33539/educacion.2014.n20.1035

De la Rosa, D., \& Mora Ramírez, Á J. (2019). Innovación educativa, nuevas metodologías y prácticas en el aula. Experiencias desde México y Colombia. Bogotá: Editorial Politécnico Grancolombiano.

Cifuentes, P. y Meseguer, P. (2015). Trabajo en equipo frente a trabajo individual: Ventajas del Aprendizaje Cooperativo en el aula de traducción. Tonos Digital (28). 17-35.

Consejo Nacional de Educación. (2006). Proyecto Educativo Nacional. Vol. 1. Lima Perú: MINEDU.

Consejo Nacional de Educación. (2020). Proyecto Educativo Nacional Al 2036. Lima, Perú: MINEDU. http://repositorio.minedu.gob.pe/handle/MINEDU/6910.

Cueto, S. (2016). Innovación y Calidad en Educación en América Latina. Primera Ed. Lima, Perú: Grambs Corporación Gráfica S.A.C. http://www2.congreso.gob.pe/sicr/cendocbib/con5_uibd.nsf/F6F6349892E56C66052582 0C0070CFE8/\$FILE/ILAIPPGRADE_innovcalEdu.pdf. 
Díaz, A. F. \& Hernández, R. G. (2015). Constructivismo y aprendizaje significativo. En: Estrategias docentes para un aprendizaje significativo, (pp. 13-33). México: Mc Graw Hill.

Gallegos, A. (1997). Lev Vygotsky: Sus aportes para el siglo XXI. Publicacio. Caracas, Venezuela: UCAB.

García-Cuevas, A., y Hernández, E. (2016). El Aprendizaje Cooperativo como estrategia para la inclusión del alumnado con Tea en el Aula Ordinaria. Revista Nacional e Internacional de Educación Inclusiva 9(2). 18-34.

García, R., Traver, J. y Candela, I. (2019). Aprendizaje Cooperativo fundamentos, características y técnicas Aprendizaje Cooperativo. Segunda ed. Madrid, España: CCS.

Guerrero, M. (2014). Metodologías activas y aprendizaje por descubrimiento. Las TIC y la Educación. Marpadal Interactive Media.

Heredia, Y. y Sánchez, A. (2020). Teorías del Aprendizaje en el contexto educativo. Monterrey México: Editorial digital del Tecnológico de Monterrey.

Herrada, R. y Baños, R. (2018). Experiencias de Aprendizaje Cooperativo en Matemáticas. Espiral. Cuadernos Del Profesorado 11(23). 99-108. http://dx.doi.org/10.25115/ecp.v11i23.2131

Huanca, O. (2017). Influencia del Aprendizaje Cooperativo en el desarrollo de los dominios en el área de matemática en los estudiantes del quinto grado de Educación Primaria En La I.E 7041 Virgen de La Merced. [Tesis doctoral, Universidad Nacional de Educación Enrique Guzmán y Valle]. http://repositorio.une.edu.pe/handle/UNE/1653

Ibarrola, B. (2014). Aprendizaje Emocionante. Neurociencia para el aula. España: Ediciones SM. Johnson, D. y Johnson, R. (2015). La Evaluación en el Aprendizaje Cooperativo. edited by E. S. España.

Johnson, D. y Johnson, R. (2014). Cooperative Learning in 21st Century. Anales de Psicología 30(3). 841-51. https://dx.doi.org/10.6018/analesps.30.3.201241

Johnson, D. y Johnson, R. (2015). Cooperative Learning and Teaching Citizenship in democracies. International Journal of Educational Research (76). 162-77. https://dx.doi.org/10.1016/j.ijer.2015.11.009.

Johnson, D., Johnson, R. y Holubec, E. (1999). El Aprendizaje Cooperativo en el aula. Argentina: Paidós.

Juárez, M., Rasskin, I. y Mendo, S. (2019). El Aprendizaje Cooperativo, Una Metodología Activa para la Educación del Siglo XXI: Una Revisión Bibliográfica. Prisma Social (26). 200210. https://revistaprismasocial.es/article/view/2693.

La Prova, A. (2017). La Práctica del Aprendizaje Cooperativo. Propuestas Operativas para el Grupo-Clase. Madrid, España: Narcea Ediciones.

Lata, S. y Castro, M. (2016). El Aprendizaje Cooperativo, un camino hacia la inclusión educativa. Revista Complutense de Educacion 27(3). 1085-1101. https://doi.org/10.5209/rev_RCED.2016.v27.n3.47441.

León, B. Felipe, E., Iglesias, D. y Marugán, M. (2014). Determinantes en la eficacia del Aprendizaje Cooperativo. Una Experiencia en el EEES. Revista de Investigacion Educativa 32(2). 411-24. https://doi.org/10.6018/rie.32.2.172721.

León, B., Felipe, E., Mendo, S. y Iglesias, D. (2015). Habilidades Sociales en equipos de aprendizaje cooperativo en el contexto universitario. Behavioral Psychology/ Psicologia Conductual 23(2). 191-214.

Lobato, C., Guerra, N. y Apodaca, P. (2015). El Aprendizaje Cooperativo En La Educación Superior: Entrenamiento En Competencias Sociales de Trabajo En Grupo. International 
Journal of Developmental and Educational Psychology. Revista INFAD de Psicología. 1(1). 377-87. http://dx.doi.org/10.17060/ijodaep.2015.n1.v1.82.

Martínez, R. y Sánchez, G. (2020). El Aprendizaje Cooperativo en la clase de Educación Física: Dificultades iniciales y propuestas para su desarrollo. Revista Educación 44(1). 2215-2644. http://dx.doi.org/10.15517/REVEDU.V44I1.35617

Mayordomo, R. y Onrubia, J. (2016). El Aprendizaje Cooperativo. $1^{\circ}$ Edición. Barcelona: Editorial UOC.

Mendo, S. (2019). Desarrollo de Habilidades Sociales en equipo en el contexto universitario: Aprendizaje Cooperativo y Entrenamiento En Habilidades Sociales. Universidad de Extremadura, Epaña.

Ministerio de Educación. (2003). Ley General de Educación $N^{\circ}$ 28044. Lima, Perú. http://www.minedu.gob.pe/p/ley_general_de_educacion_28044.pdf.

Ministerio de Educación. (2016). Currículo Nacional de la Educación Básica. Lima, Perú: MINEDU. http://www.minedu.gob.pe/curriculo/pdf/curriculo-nacional-de-la-educacionbasica.pdf.

Ministerio de Educación. (2018). Marco del buen desempeño docente. Lima, Perú: MINEDU. http://www.minedu.gob.pe/pdf/ed/marco-de-buen-desempeno-docente.pdf.

Morales, L., García, O., Torres, A. y Lebrija, A. (2018). Habilidades cognitivas a través de la estrategia de aprendizaje cooperativo y perfeccionamiento epistemológico en matemática de estudiantes de primer año de universidad. Formación Universitaria 11(2). 45-56. http://dx.doi.org/10.4067/S0718-50062018000200045

Naciones Unidas/CEPAL. (2018). La Agenda 2030 y Los Objetivos de Desarrollo Sostenible: Una Oportunidad Para América Latina y El Caribe. Objetivos, Metas e Indicadores Mundiales. Santiago: Naciones unidas.

Olmedo, N. y Farrerons, O. (2017). Modelos constructivistas de aprendizaje en programas de formación. Catalunya España: OmniaScience. https://doi.org/10.3926/oms.367

Pevida, M. y Gallego, A. (2014). Compartir para aprender y aprender a cooperar. Vol. 369. Oviedo-España.

Robles, L. (2015). El Trabajo Cooperativo. Revista Internacional de Apoyo a La Inclusión, Logopedia, Sociedad y Multiculturalidad 1(2). 57-66.

Rodríguez, M. y Barragán, H. (2017). Entornos Virtuales de Aprendizaje como apoyo a la enseñanza presencial para potenciar el proceso educativo. Revista Killkana Sociales 1(2). 7-14. https://doi.org/10.26871/killkana_social.v1i2.29.

Sáez, J. (2018). Estilos de Aprendizaje y Métodos de Enseñanza. Madrid España: UNED.

Sánchez, I. y Casal, S. (2016). El Desarrollo de la autonomía mediante las técnicas de Aprendizaje Cooperativo En El Aula de L2. Porta Linguarum 25(1). 179-90. https://doi.org/10.30827/Digibug.53915

Serrano, J. y Pons, R. (2014). Introduction: Cooperative Learning. Anales de Psicología 30(3). 781-84. http://dx.doi.org/10.6018/analesps.30.3.201251

Slavin, R. (1999). Aprendizaje Cooperativo: Teoría, Investigacióny Práctica. Aique Grupo Editor S.R.L.

Soto, J. (2017). Relación Del aprendizaje cooperativo y los estilos de aprendizaje con el rendimiento académico del área de ciencia tecnología y ambiente de los estudiantes de la I.E. Tungasuca de Carabayllo, 2016. [Tesis de doctorado, Universidad César Vallejo Perú]. https://hdl.handle.net/20.500.12692/8479

Torrego, J., Boal, M., Bueno, A., Calvo, E., Monserrat, M., Maillo, I., Miguel, A., Moruno, P., 
Moya, A., Rodríguez, A., Rodríguez, G., Ruiz, V., Sánchez, M., Torrego, Y., Varas, M., Vega, S. y Zariquiey, F. (2011). Alumnos con altas capacidades y Aprendizaje Cooperativo. Un modelo de respuesta educativa. Madrid, España: Fundación SM.

Torres, M. (2017). Metodología Cooperativa utilizando las tecnologías de la información y comunicación en el aprendizaje del idioma inglés En Los estudiantes de tercer nivel de la Facultad de Ingeniería - Universidad Nacional de Chimborazo Riobamba Ecuador. Universidad Nacional de Chimborazo, Ecuador.

Zariquiey, F. (2016). Cooperar para aprender. Transformar el aula en una red de Aprendizaje Cooperativo. España: Ediciones SM. 